\title{
COHEN-MACAULAYNESS OF BLOW-UPS OF HOMOGENEOUS WEAK $d$-SEQUENCES
}

\author{
MARK R. JOHNSON AND K. N. RAGHAVAN \\ (Communicated by Wolmer V. Vasconcelos)
}

\begin{abstract}
Let $R$ be a homogeneous Cohen-Macaulay algebra over a field, and let $I$ be an ideal generated by a homogeneous weak $d$-sequence. We show, under reasonable conditions on the sequence, that the graded ring $\operatorname{gr}_{M} R[I t]$ of the Rees algebra $R[I t]=\bigoplus_{i>0} I^{i}$ is Cohen-Macaulay. In particular we obtain the Cohen-Macaulayness of the blow-up ring $R[I t]$.
\end{abstract}

In this note we prove a result about the Cohen-Macaulayness of the blowup rings of certain homogeneous ideals generated by weak $d$-sequences with good depth properties. It is a generalization, in the graded case, of well-known results about the Cohen-Macaulayness of blow-up rings of ideals generated by $d$-sequences, proved by Huneke ([H1]) and Herzog-Simis-Vasconcelos ([HSV]). Huneke later introduced weak $d$-sequences in [H2] to study depths of powers of ideals of various determinantal varieties.

By making use of a sort of Gröbner basis technique following [HTU] and [RS], we filter the ring $\operatorname{gr}_{M}(R[I t])$ and show the ideal of initial forms is CohenMacaulay via the standard exact sequence $0 \rightarrow K_{1} \cap K_{2} \rightarrow K_{1} \oplus K_{2} \rightarrow K_{1}+K_{2} \rightarrow$ 0 . The ideals which occur here involve essentially the related ideals and the initial quadratic relations of the given sequence.

Before stating the result, we will need some definitions. First we recall the definition of a quadratic sequence, which is a slight generalization of a weak $d$-sequence ([R]). Let $H$ be a partially ordered set and $A \subset H$ an $H$-ideal (i.e. $A$ is closed under <). If $\alpha \in H$ and lies just above $A$, i.e. $\beta<\alpha \Rightarrow \beta \in A$, we say $(A, \alpha)$ is a pair. Now let $\left\{x_{\alpha}\right\}_{\alpha \in H} \subset R$ be elements of a Noetherian ring $R$ indexed by $H$. Let $I_{A}=\left(x_{\alpha} \mid \alpha \in A\right)$ and $I=I_{H}$. The sequence $\left\{x_{\alpha}\right\}$ is a quadratic sequence if for every pair $(A, \alpha)$ of $H$, there exists an $H$-ideal $B$ so that $I_{A}: x_{\alpha} \cap I=I_{B}$ and $x_{\alpha} I_{B} \subset I_{A} I$.

In an attempt to mimic a $d$-sequence situation, which is linear, we consider a linearization of the weak $d$-sequence. By definition, this is just a bijective map of partially ordered sets $H \rightarrow\{1,2, \ldots, n\}$. We will fix this linearization, once and for all, and define everything else with respect to this. We now write $x_{1}, \ldots, x_{n}$ for the linearized quadratic sequence. In the homogeneous case we

Received by the editors November 16, 1993.

1991 Mathematics Subject Classification. Primary 13A30. 
require that this map be degree preserving, which just means that $\operatorname{deg} x_{1} \leq \cdots \leq$ $\operatorname{deg} x_{n}$.

The related ideals $I_{j}$, for $j=1, \ldots, n$, are the colon ideals

$$
I_{j}=\left(x_{1}, \ldots, x_{j-1}\right): x_{j} .
$$

In the polynomial ring $k\left[T_{1}, \ldots, T_{n}\right]$ the initial quadratic relations are defined by

$$
Q=\left(T_{j} T_{k} \mid x_{k} \in I_{j}, \quad 1 \leq j \leq k \leq n\right) .
$$

The name will be justified shortly. We say the sequence is squarefree if $Q$ is generated by squarefree monomials.

We call a linearized and degree-preserving quadratic sequence standard if it satisfies the condition

$$
x_{k} \notin I_{j} \Rightarrow I_{j} \subset I_{k} \text {. }
$$

Note that any $d$-sequence is standard since $I_{1} \subset \cdots \subset I_{n}$; so is any straightening closed ideal in an ordinal Hodge algebra ([BST]).

Finally we will need the auxiliary ideals

$$
Q_{k}=\left(T_{j} T_{l} \in Q \mid 1 \leq j \leq l<k\right)
$$

and

$$
Q_{k}^{\prime}=Q_{k}+\left(T_{j} \mid T_{j} T_{k} \in Q, j<k\right)+\left(T_{k}^{2} \mid T_{k}^{2} \in Q\right) .
$$

Now let $S=R\left[T_{1}, \ldots, T_{n}\right]$ be the polynomial ring over $R$. Recall the degree-lexicographic order on $N_{0}^{n+1}$ is defined by $\left(\alpha_{0}, \ldots, \alpha_{n}\right)<\left(\beta_{0}, \ldots, \beta_{n}\right)$ if the first nonzero component from the left side of

$$
\left(\sum_{i=0}^{n} \alpha_{i}-\sum_{i=0}^{n} \beta_{i}, \alpha_{1} \beta_{1}, \ldots, \alpha_{n}-\beta_{n}\right)
$$

is negative. Think of $S=\bigoplus S_{h}$ as $N_{0}^{n+1}$ graded. If we set $F_{h} S=\bigoplus_{g \geq h} S_{g}$, then $F=\left\{F_{h} S\right\}_{h \in N_{0}^{n+1}}$ is a filtration on $S$ finer than its $M$-adic filtration ( $M$ is the irrelevant maximal ideal).

We have $\operatorname{gr}_{F}(R[I t])=\operatorname{gr}_{F}(S / J) \cong S / J_{*}$, where $J_{*}$ is the ideal generated by the initial forms of elements of $J$. Under a slightly technical stability condition on the linearization, one has ([RS, Theorem 1.4])

$$
J_{*}=I_{1} T_{1}+\cdots+I_{n} T_{n}+Q .
$$

Theorem. Let $R$ be a homogeneous Cohen-Macaulay algebra over a field, and let $I$ be an $R$-ideal generated by a stable, standard homogeneous quadratic sequence with $I_{1}=0$. If for $j=1, \ldots, n$

$$
\operatorname{depth} S /\left(I_{j}, Q_{j}^{\prime}\right) \geq \operatorname{dim} S-j+1,
$$

then $\operatorname{gr}_{M} R[I t]$ is a Cohen-Macaulay ring.

Corollary ([HTU, Theorem 1.6]). Let $R$ be a Cohen-Macaulay homogenous algebra over a field, and $I$ an ideal generated by a homogeneous $d$-sequence $x_{1}, \ldots, x_{n}$ with $\operatorname{deg} x_{1} \leq \cdots \leq \operatorname{deg} x_{n}$. If grade $I>0$ and $\operatorname{depth} R / I_{j}=$ $\operatorname{dim} R-j+1$ for $j=1, \ldots, n$, then $g r_{M} R[I t]$ is Cohen-Macaulay.

Proof. Since $x_{1}, \ldots, x_{n}$ is a $d$-sequence, it is a stable, linearized degreepreserving quadratic sequence, $Q=0$ and $I_{1}=0: x_{1}=0: I=0$ since grade $I>0$.

It is not hard to check that a squarefree, standard weak $d$-sequence is stable. Hence one has the following 
Corollary. Let $R$ be a homogeneous Cohen-Macaulay algebra over a field, and let $I$ be an ideal generated by a standard, squarefree homogeneous weak $d$ sequence with $I_{1}=0$. If for $j=1, \ldots, n$, depth $S /\left(I_{j}+Q_{j}^{\prime}\right) \geq \operatorname{dim} S-j+1$, then $R[I t]$ is a Cohen-Macaulay algebra.

Proof of Theorem. It is enough to show that $S / J_{*}$ is Cohen-Macaulay.

Consider

$$
J_{*}=I_{1} T_{1}+\cdots+I_{n} T_{n}+Q \subset S=R\left[T_{1}, \ldots, T_{n}\right] .
$$

Write

$$
J_{*}=L_{1} T_{1}+\cdots+L_{n} T_{n}
$$

where $L_{k}=I_{k}+\left(T_{j} \mid T_{j} T_{k} \in Q, j \leq k\right)$.

We will show, by induction on $k$, that $\operatorname{depth} S / J_{k} \geq \operatorname{dim} S-k+1$, where $J_{k}=L_{1} T_{1}+\cdots+L_{k} T_{k}$. This will prove the result since then depth $S / J_{*}=$ $\operatorname{depth} S / J_{n} \geq \operatorname{dim} S-n+1=\operatorname{dim} R+1=\operatorname{dim} S / J_{*}$ and hence $S / J_{*}$ is CohenMacaulay.

If $k=1$, then $J_{1}=L_{1} T_{1}=0$ since $I_{1}=0$ and so $\operatorname{depth} S / J_{1}=\operatorname{dim} S$. Now suppose $k \geq 2$ and that by induction we have shown $\operatorname{depth} S / J_{k-1} \geq$ $\operatorname{dim} S-k+2$.

Claim. $J_{k}=\left(J_{k-1}, T_{k}\right) \cap\left(I_{k}, Q_{k}^{\prime}\right)$.

Given the claim, we have an exact sequence

$$
0 \rightarrow S / J_{k} \rightarrow S /\left(J_{k-1}, T_{k}\right) \oplus S /\left(I_{k}, Q_{k}^{\prime}\right) \rightarrow S /\left(I_{k}, Q_{k}^{\prime}, T_{k}\right) \rightarrow 0 .
$$

Since by assumption depth $S /\left(I_{k}+Q_{k}^{\prime}\right) \geq \operatorname{dim} S-k+1$, by induction we have depth $S / J_{k} \geq \operatorname{dim} S-k+1$ as required.

Proof of Claim. (C) Suppose some $L_{i} T_{i}$ is not contained in $\left(I_{k}, Q_{k}^{\prime}\right)$, for some $i, 1 \leq i \leq k$. By definition of $Q_{k}^{\prime}, I_{i}$ is not contained in $I_{k}$. Since $I$ is standard, there is a quadratic relation $T_{i} T_{k} \in Q$, hence $T_{i} \in Q_{k}^{\prime}$. This would imply $L_{i} T_{i} \subset Q_{k}^{\prime}$, which is a contradiction.

(כ) It's enough to see that an element $a T_{k} \in\left(I_{k}, Q_{k}^{\prime}\right)$ must lie in $J_{k}$. Consider two cases. First if $T_{k}^{2} \notin Q$, then this is clear since then $a \in\left(I_{k}, Q_{k}^{\prime}\right)$, as $Q_{k}^{\prime}$ contains no monomials involving $T_{k}$, and in the second case if $T_{k}^{2} \in Q$, then $a \in\left(I_{k}, Q_{k}, T_{k}\right)$. In either case, $a T_{k} \in J_{k}$. This proves the claim and the theorem.

Remark. Actually the proof shows that even more is true. Let $\pi$ be a permutation of $\{1,2, \ldots, n\}$ and $I$ a (stable) linearized degree-preserving homogeneous quadratic sequence with $I_{1}=0$ (not necessarily standard).

Call the sequence $\pi$-standard if $x_{\pi(i)} \notin I_{\pi(j)} \Rightarrow I_{\pi(i)} \subset L_{\pi(j)}$ and also

$$
x_{l} \in I_{\pi(i)}, 1 \leq l \leq \pi(i) \Rightarrow l=\pi(k), 1 \leq k \leq j-1 .
$$

(If $\pi$ is the identity permutation, then $\pi$-standard is the same as standard.) Put $Q_{k}^{\pi}=\left(T_{j} T_{l} \in Q \mid j, l \in \pi(\{1, \ldots, k-1\})\right)$ and $Q_{k}^{\pi^{\prime}}=Q_{k}^{\pi}+\left(T_{j} \mid T_{j} T_{\pi(k)} \in\right.$ $Q, j \in \pi(\{1, \ldots, k-1\}))+\left(T_{\pi(k)}^{2} \in Q\right)$.

Then if the sequence if $\pi$-standard and satisfies depth $S /\left(I_{\pi(j)}+Q_{j}^{\prime}\right) \geq \operatorname{dim} S-$ $j+1$, then $\mathrm{gr}_{M} R[I t]$ is Cohen-Macaulay.

One just applies the permutation $\pi$ to the $L$ 's in the proof.

We conclude by mentioning two cases where the theorem readily applies. This gives very different proofs of these facts. 
Example 1. Straightening closed ideals in an ordinal Hodge Algebra. In [EH] the Rees algebras of such ideals was shown to be Cohen-Macaulay. Indeed, these are always standard, squarefree weak $d$-sequences satisfying our depth condition. In particular this includes the fact that $R\left[I_{n}(X) t\right]$ is Cohen-Macaulay for $R=k[X], X$ a generic $n$ by $m$ matrix of variables. (See [EH] and [BST] for more details.)

Example 2. Monomial curves in $P^{3}$ on a quadric. These are the irreducible rational curves $C$ parametrized by $s^{d}, s^{a} t^{d-a}, s^{b} t^{d-b}, t^{d}$. Let $I=I(C)$ be its homogeneous ideal in $R=k\left[x_{0}, \ldots, x_{3}\right]$. The case that $C$ lies on a quadric has been recently considered in its context. Note that if it lies on a cone, it must be arithmetically Cohen-Macaulay and hence $I$ is generated by a $d$-sequence of 3 elements. So we may it assume it lies on the hypersurface $x_{0} x_{3}=x_{1} x_{2}$. $R[I t]$ was shown to be Cohen-Macaulay in [S], [MS], and [HH]. In the first two works, the entire defining ideal of $R[I t]$ had to be explicitly computed, while in the third one had to first establish that $I$ is normally torsion free. As is well known (cf., e.g., [MS]), $I$ is generated by a weak $d$-sequence, and the generators can be linearly ordered to give $I_{2}=\left(x_{0} x_{3}-x_{1} x_{2}\right), I_{j}=\left(x_{0}, x_{1}\right)$, $3 \leq j \leq n, Q=\left(T_{3}, \ldots, T_{n-1}\right)^{2}$. The sequence was checked stable in [RS]. Applying the permutation $\{1,2, \ldots, n\} \rightarrow\{1,2, n, n-1, \ldots, 3\}$, by the remark $\operatorname{gr}_{M}(R[I(C) t])$ is Cohen-Macaulay.

\section{REFERENCES}

[BST] W. Bruns, A. Simis, and N. V. Trung, Blow-up of straightening-closed ideals in ordinal hodge algebras, Trans. Amer. Math. Soc. 326 (1991), 507-528.

[EH] D. Eisenbud and C. Huneke, Cohen-Macaulay Rees algebras and their specializations, J. Algebra 81 (1983), 202-224.

[HSV] J. Herzog, A. Simis, and W. V. Vasconcelos, Koszul homology and blowing up rings, Lecture Notes in Pure and Appl. Math., vol. 84, Marcel Dekker, New York, 1983, pp. 79-169.

[HH] S. Huckaba and C. Huneke, Powers of ideals having small analytic deviation, Amer. J. Math. 114 (1992), 367-403.

[H1] C. Huneke, Symbolic powers of prime ideals and special graded algebras, Comm. Algebra 9 (1981), 339-366.

[H2] _ Powers of ideals generated by weak d-sequences, J. Algebra 68 (1981), 471-509.

[HTU] J. Herzog, N. V. Trung, and B. Ulrich, On the multiplicity of blow-up rings of ideals generated by d-sequences, J. Pure Appl. Algebra 80 (1992), 273-297.

[MS] M. Morales and A. Simis, Symbolic powers of monomial curves in $\mathrm{P}_{3}$ lying on $x w-y z=0$, Comm. Algebra 20 (1992), 1109-1121.

[R] K. Raghavan, Powers of ideals generated by quadratic sequences, Trans. Amer. Math. Soc. 343 (1994), 727-747.

[RS] K. Raghavan and A. Simis, Multiplicities of blow-ups of homogeneous quadratic sequences, preprint.

[S] P. Schenzel, Examples of Gorenstein domains and symbolic powers of monomial space curves, J. Pure Appl. Algebra 71 (1991), 297-311. 48824

Current address, K. N. Raghavan: School of Mathematics, SPIC Science Foundation, East Coast Chambers 4th floor, 92 G. N. Chetty Road, T. Nagar, Madras 600017, India 